\title{
Study Of The Relationship Between Dielectric Properties Of Indian Jujube
}

\author{
Yu-Kai Weng ${ }^{1}$, Ching-Wei Cheng ${ }^{2}$, Chia-Chung Chen ${ }^{1}$, Cheng-Chu Hung ${ }^{1}$, Cheng-Han Li ${ }^{1}$ \\ ${ }^{1}$ Department of Bio-industrial Mechatronics Engineering/National Chung Hsing University \\ No.145 Xingda Rd., South Dist., Taichung City 402, Taiwan (R.O.C.) \\ ykweng@dragon.nchu.edu.tw \\ ${ }^{2}$ College of Intelligence/National Taichung University of Science and Technology \\ No.129, Sec.3, Sanmin Rd., North Dist., Taichung City 404, Taiwan (R.O.C.)
}

\section{Extended Abstract}

The methods to distinguish the maturity of fruits are based on the experienced of farmers; different kinds of fruits require different techniques. The evaluation methods include color, hardness, and sound to identify maturity. However, the results obtained based on experience may not be matched to the quality of the fruit; also, manually will pay more time-cost. The dielectric permittivity measurement of fruits is based on that the electrical parameters of fruits in the electric field will change with the frequency of the electric field. And the use of changes in electrical characteristics and destructive testing of fruits include: moisture content, firmness, sugar content, and $\mathrm{PH}$ value and find the correlation between the two to determine the quality of the fruit.

For example, Measuring reactance $(\mathrm{X})$, resistance $(\mathrm{R})$, capacitance $(\mathrm{C})$ and impedance $(\mathrm{Z})$ as a function of frequency. [1] The biological impedance of the banana is measured in the frequency range under different ripening states. The ripening state is correlated with its corresponding impedance, thereby providing an estimate of the fruit substance content. Measure and study the bio-impedance parameters of fruit at a continuously changing frequency to study the frequency-dependent behavior of fruit ripening. [2]

The results indicated that the relationship between soluble solids content and moisture are negatively correlated. The higher the maturity, the higher the sugar content and gradually lose water as the storage time increases.

The hardness decreases with the increase of storage days, but it increases on the 10th day. The reason is that the fruit's toughness of the epidermis increases. It will absorb the force applied to the jujube, lead to sunken when using the hardness tester without damaging the peel, but it will break when it reaches a particular strength.

The Nyquist diagram is the real and imaginary parts of the impedance obtained at fixed frequency intervals (frequency values increase from right to left). Indicates that it is greatly affected by the low-frequency region because compared. With the increase of storage days, the complex impedance value also gradually increases. Speculate that due to the loss of water and the rise of soluble solid content will cause electrical impedance increase.

The reason for the increase in maturity might be the loss of water and the rise of soluble solid content or other physiological changes. The decrease in ions in banana tissue causes by decreasing electrical conductivity. The decrease in electrical conductivity, the increase in impedance. The Nyquist diagram obtained by maturity shows the obvious deviation of the real part impedance data and the imaginary part impedance data. [3] The main reason for the change of fruit impedance during storage is its moisture and the relative concentration of charged particles. During storage, the increase in mineral concentration may be due to evapotranspiration. Evapotranspiration is caused by the evaporation of water in the tissue, and its rate depends on internal factors. Therefore, we can consider the loss of water caused by evapotranspiration and the increase in ion concentration as the main factors for the change of equivalent circuit parameters. [4]

Establish test systems capable of measuring the electrical characteristics of jujubes. The frequency characteristics of the equivalent impedance, relative permittivity, loss factor, and conductance of the jujubes were measured through using a parallel plate dielectric in the frequency range of $1 \mathrm{kHz} \sim 300 \mathrm{kHz}$. From the Nyquist diagram, it is feasible to evaluate the internal quality of fruits from the impedance characteristics of jujubes.

\section{References}

[1] Atanu C., D. Ghoshal and B. Chakraborty. 2016. Electrical Impedance Variations in Banana Ripening: An Analytical Study with Electrical Impedance Spectroscopy. Journal of Food Process Engineering. 40(2). 
[2] N. V. Mane and R. P. Mudhalwadkar. 2017. Banana ripeness assessment by impedance spectroscopy. 2017 IEEE International Conference on Power, Control, Signals and Instrumentation Engineering (ICPCSI): pp. 527-529.

[3] Atanu C., D. Ghoshal and B. Chakraborty. 2016. Electrical Impedance Variations in Banana Ripening: An Analytical Study with Electrical Impedance Spectroscopy. Journal of Food Process Engineering. 40(2).

[4] Pietro I., A. Falco, B. D. Abera, G. Cantarella, L. Petti and P. Lugli. 2020. Bio-impedance and circuit parameters: An analysis for tracking fruit ripening. Postharvest Biology and Technology. 159: 110978. 